\title{
Psychological and behavioral approaches to cardiac patients facing specific adjustment challenges
}

\author{
Gian M. Manzoni ${ }^{1,2 *}$, Giada Pietrabissa ${ }^{1,2}$ and Gianluca Castelnuovo ${ }^{1,2}$ \\ ${ }^{1}$ Psychology Research Laboratory, Istituto Auxologico Italiano IRCCS, Ospedale San Giuseppe, Verbania, Italy \\ 2 Department of Psychology, Catholic University of Milan, Milan, Italy \\ *Correspondence: gm.manzoni@auxologico.it \\ Edited by: \\ Francesco Pagnini, Catholic University of Milan, Italy \\ Reviewed by: \\ Chiara A. M. Spatola, Istituto Auxologico Italiano, Italy
}

\begin{abstract}
A book review on
Stress Proof the Heart. Behavioral Interventions for Cardiac Patients

Edited by Ellen A. Dornellas, Springer Science+Business Media, LLC 2012, 400 pages. ISBN: 978-1-4419-5649-1; e-ISBN 978-1-44195650-7
\end{abstract}

To some extent, all cardiac patients are challenged by their heart disease. Challenging issues concern the adjustment of patients to the cardiovascular disease and involve the physical, psychological, behavioral, and social dimensions of the person. Clearly, heart disease, its physical impact and the clinical sequelae (treatment and rehabilitation procedures) challenge patients at multiple levels by critically upsetting the homeostasis of their bio-psycho-social systems. Challenges may be very easy as well as very hard to accomplish and this variability does not depend only on the seriousness of the cardiac illness or on the impact of the correlated clinical and rehabilitation procedures, but it is also related to the psychosocial characteristics of patients, including personality and coping styles. When patients have difficulties in facing the multi-dimensional challenges of heart disease, they may show high levels of stress, anxiety, and depression as well as problematic behaviors such as resistance and poor adherence to treatment and preventive prescriptions, including behavioral modifications such as smoking cessation and physical exercise improvement.

Given the huge amount of empirical evidence supporting the fact that smoking, physical inactivity, unhealthy diet, harmful use of alcohol as well as psychological distress are modifiable risk factors for cardiovascular morbidity and mortality, patients showing difficulties in adjusting to cardiac illness and the correlated clinical demands should be detected and then helped with behavioral and psychological interventions in facing the multidimensional challenges they are not able to overcome themselves.

This book, edited by an experienced health psychologist in the field of "behavioral cardiology" and written by an international group of outstanding researchers and clinicians working in the expanding area of bio-psycho-social approaches to cardiac patients, covers firstly the psychological challenges and management needs of patients affected by specific cardiovascular conditions. With few exceptions, all chapters in the first part deserve particular consideration for their specialty contents. Clinicians working with cardiac patients affected by specific cardiovascular conditions such as ICD implantation for ventricular fibrillation, atrial fibrillation, heart failure, Takotsubo syndrome, and cardiac transplantation, may find in them extensive information about the unique psychological challenges and difficulties of living with such conditions and they may also acquire specialty knowledge on tailored psychological approaches to specific needs management.

In the second and last part of the book, various psychological and behavioral interventions for cardiac patients are described. With the exception of the review chapter about anxiety and depression as risk factors for cardiovascular disease, whose inclusion in this section is questionable, the great part of issues that are covered along the second half of this book were rarely or never addressed in other similar publications. Bright spots are the chapters on computerized therapies and on the management of sleep problems, while a missing issue is the psychological and behavioral management of obese patients with heart disease, for which tailored multi-disciplinary interventions have already been planned and assessed (Manzoni et al., 2010, 2011).

The declared purpose of this book is to inform clinicians who practice on the front line of the psychological and behavioral management of cardiac patients on topics where the research findings have not yet caught with practice and, at the same time, to provide them concrete, immediately applicable, translation of clinical research findings into tailored practical approaches (Castelnuovo, 2010a,b). It is noteworthy that clinicians whose this book is addressed will find in it all that was promised.

\section{REFERENCES}

Castelnuovo, G. (2010a). Empirically supported treatments in psychotherapy: towards an evidence-based or evidence-biased psychology in clinical settings? Front. Psychology 1:27. doi: 10.3389/fpsyg.2010.00027

Castelnuovo, G. (2010b). No medicine without psychology: the key role of psychological contribution in clinical settings. Front. Psychology 1:4. doi: 10.3389/fpsyg.2010.00004 
Manzoni, G. M., Cribbie, R. A., Villa, V., Arpin-Cribbie, C. A., Gondoni, L., and Castelnuovo, G. (2010). Psychological wellbeing in obese inpatients with ischemic heart disease at entry and at discharge from a four-week cardiac rehabilitation program. Front. Psychology 1:38. doi: 10.3389/fpsyg.2010. 00038

Manzoni, G. M., Villa, V., Compare, A., Castelnuovo, G., Nibbio, F., Titon, A. M., et al. (2011). Shortterm effects of a multi-disciplinary cardiac rehabilitation programme on psychological wellbeing, exercise capacity and weight in a sample of obese in-patients with coronary heart disease: a practice-level study. Psychol. Health Med. 16, 178-189.

Received: 17 September 2012; accepted: 16 October 2012; published online: 01 November 2012.

Citation: Manzoni GM, Pietrabissa G and Castelnuovo $G$ (2012) Psychological and behavioral approaches to cardiac patients facing specific adjustment challenges. Front. Psychology 3:476. doi: 10.3389/fpsyg.2012.00476 This article was submitted to Frontiers in Psychology for Clinical Settings, a specialty of Frontiers in Psychology. Copyright (c) 2012 Manzoni, Pietrabissa and Castelnuovo. This is an open-access article distributed under the terms of the Creative Commons Attribution License, which permits use, distribution and reproduction in other forums, provided the original authors and source are credited and subject to any copyright notices concerning any third-party graphics etc. 